\title{
Dupilumab in Adults with Moderate-to-Severe Atopic Dermatitis and Prior Use of Systemic Non-Steroidal Immunosuppressants: Analysis of Four Phase 3 Trials
}

\author{
Christopher Griffiths (1) - Marjolein de Bruin-Weller (1) - Mette Deleuran (1) - Maria Concetta Fargnoli (1) · \\ Delphine Staumont-Sallé (1) · Chih-ho Hong (1) · Jose Sánchez-Carazo (1) · Peter Foley (1) · Seong Jun Seo (1) · \\ Jérôme Msihid (1) · Zhen Chen · Sonya L. Cyr (i) · Ana B. Rossi (1)
}

Received: March 31, 2021 / Accepted: May 24, 2021 / Published online: June 18, 2021

(c) The Author(s) 2021, corrected publication 2021

\section{ABSTRACT}

Introduction: Dupilumab is approved as firstline systemic treatment for adults/adolescents

Supplementary Information The online version contains supplementary material available at https:// doi.org/10.1007/s13555-021-00558-0.

\section{Griffiths}

The Dermatology Centre, NIHR Manchester

Biomedical Research Centre, Salford Royal Hospital,

University of Manchester, Manchester, UK

M. de Bruin-Weller

Department of Dermatology and Allergology,

National Expertise Center of Atopic Dermatitis,

University Medical Center, Utrecht, Netherlands

\section{Deleuran}

Department of Dermatology, Aarhus University

Hospital, Aarhus, Denmark

\author{
M. C. Fargnoli \\ Dermatology, Department of Biotechnological and \\ Applied Clinical Sciences, University of L'Aquila, \\ L'Aquila, Italy \\ D. Staumont-Sallé \\ CHU de Lille, Service de Dermatologie, University of \\ Lille, Lille, France \\ C. Hong \\ Department of Dermatology and Skin Science, \\ University of British Columbia, Surrey, BC, Canada \\ C. Hong \\ Probity Medical Research, Waterloo, ON, Canada
}

with moderate-to-severe atopic dermatitis (AD) in Europe and elsewhere owing to its favourable benefit-risk profile. However, systemic nonsteroidal immunosuppressants (NSISS) are often used as first-line therapy in clinical practice. Impact of prior therapy with NSISS on dupilumab's treatment effect vs. control has not been described previously. This study assessed

\author{
J. Sánchez-Carazo \\ Hospital General Universitario de València, \\ València, Spain \\ P. Foley \\ Department of Medicine, St. Vincent's Hospital \\ Melbourne, The University of Melbourne, Parkville, \\ Australia \\ P. Foley \\ Probity Medical Research, Skin Health Institute, \\ Carlton, VIC, Australia \\ S. J. Seo \\ Chung-Ang University Hospital, Seoul, South Korea \\ J. Msihid \\ Sanof, Chilly-Mazarin, France \\ Z. Chen · S. L. Cyr \\ Regeneron Pharmaceuticals, Inc., Tarrytown, NY, \\ USA \\ A. B. Rossi $(\bowtie)$ \\ Sanofi Genzyme, Cambridge, MA, USA \\ e-mail: Ana.Rossi@sanofi.com
}


dupilumab's efficacy vs. control in patients with moderate-to-severe $\mathrm{AD}$, comparing treatment effect in patients with/without prior systemic NSISS therapy, in four phase 3 trials.

Methods: This post hoc analysis included 1553 patients randomized to placebo or dupilumab (300 mg q2w) as monotherapy for 16 weeks, or with concomitant topical corticosteroids (TCS) for $16 / 52$ weeks, from four randomized, doubleblind, placebo-controlled, phase 3 trials. Patients were stratified by prior use of systemic NSISS and dupilumab-treated patients were analysed against control groups (treated with placebo or placebo + TCS).

Results: Dupilumab-treated patients, regardless of prior treatment with NSISS, achieved a significantly higher percentage reduction from baseline in Eczema Area and Severity Index (EASI), SCORing Atopic Dermatitis (SCORAD), Dermatology life Quality Index (DLQI), and Patient-Oriented Eczema Measure (POEM) vs. control; significantly more achieved EASI score $\leq 7$, Peak Pruritus Numerical Rating Scale $\leq 4$, POEM $\leq 7$, and DLQI $\leq 5$ by week 4 . These rapid, significant improvements were seen with or without concomitant TCS and sustained through end-of-treatment.

Conclusions: Dupilumab treatment (monotherapy or + TCS) provides rapid, significant, sustained improvements in signs, symptoms, and quality of life in patients with moderate-to-severe $\mathrm{AD}$ compared with control, regardless of prior systemic NSISS use.

Clinical Trial Registration: LIBERTY AD SOLO 1: ClinicalTrials.gov identifier NCT02277743, EudraCT 2014-001198-15. LIBERTY AD SOLO 2: ClinicalTrials.gov identifier NCT02277769, EudraCT 2014-002619-40. LIBERTY AD CHRONOS: ClinicalTrials.gov identifier NCT02260986, EudraCT 2013-003254-24. LIBERTY AD CAFÉ: ClinicalTrials.gov identifier NCT02755649, EudraCT 2015-002653-35. 


\section{Graphic Abstract:}

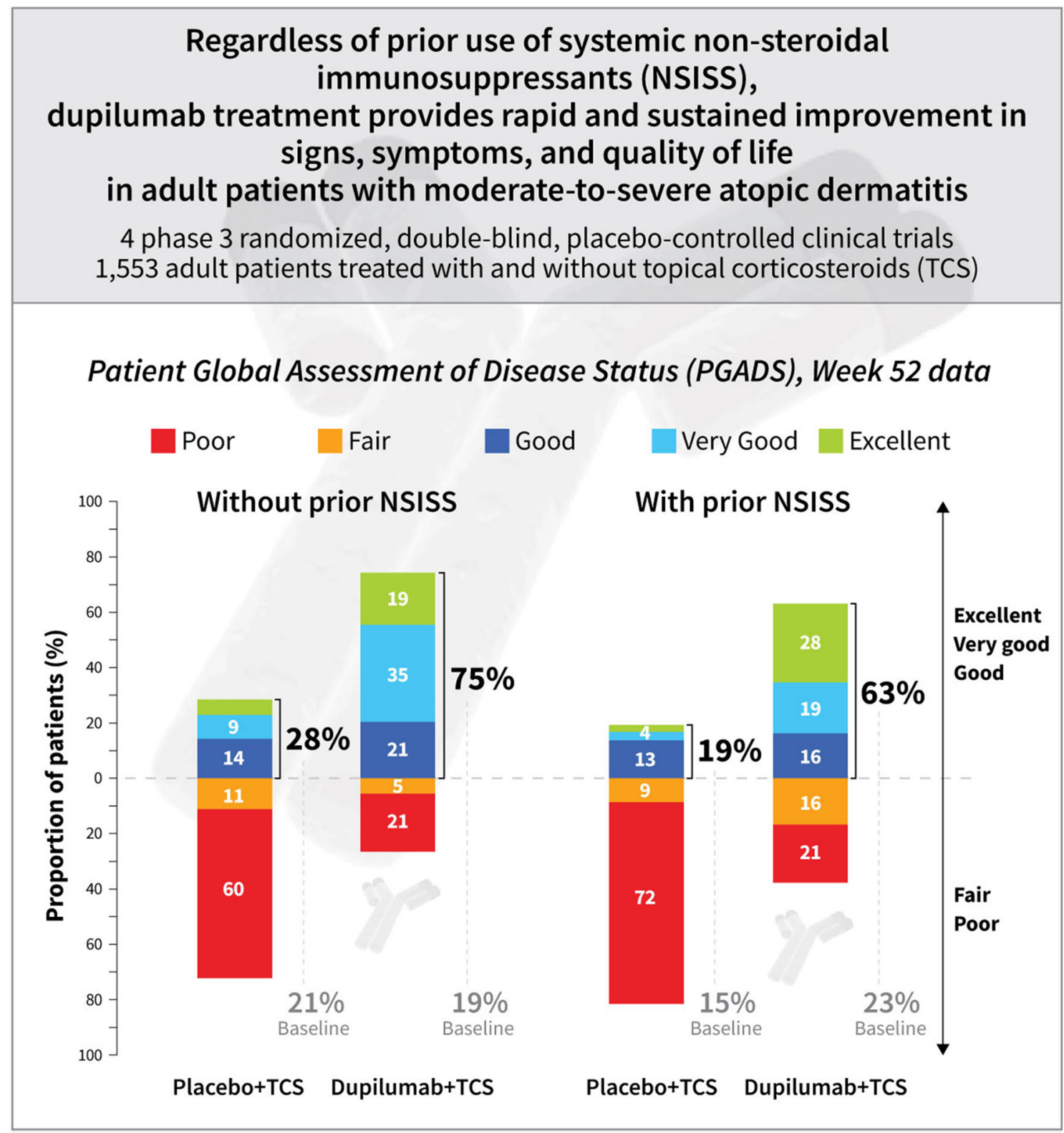

DUPILUMAB IN ADULTS WITH MODERATE-TO-SEVERE ATOPIC DERMATITIS AND PRIOR USE OF SYSTEMIC NON-STEROIDAL-IMMUNOSUPPRESSANTS: ANALYSIS OF FOUR PHASE 3 TRIALS

Christopher Griffiths, Marjolein de Bruin-Weller, Mette Deleuran, Maria Concetta Fargnoli, Delphine Staumont-Salle, Chih-ho Hong, Jose Sanchez-Carazo, Peter Foley, Seong Jun Seo, Jérôme Msihid, Zhen Chen, Sonya L. Cyr, Ana B. Rossi

The graphical abstract represents the opinions of the authors. For a full list of declarations, including funding and author disclosure statements, please see the full text online. (C) The authors, CC-BY-NC 2021.

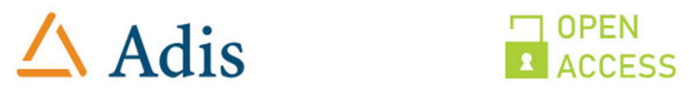




\section{PLAIN LANGUAGE SUMMARY}

Atopic dermatitis (AD), also known as eczema, is characterized by red, oozy, and dry skin that can become cracked and infected. Dupilumab is a drug that blocks key molecules that cause allergic conditions, such as AD. It has been shown to be effective in treating moderate-tosevere $\mathrm{AD}$. Other drugs commonly used to treat AD include certain anti-inflammatory drugs, known as non-steroidal immunosuppressants (NSISS), such as cyclosporin. It is not known if patients treated in the past with NSISS get the same results from AD treatment with dupilumab. This analysis used data from four large studies that included patients with moderateto-severe $\mathrm{AD}$. The objective was to see if prior NSISS use impacted how dupilumab worked to control AD. The researchers looked at a range of measurements-including ones that were assessed by a patient's doctor such as measurements of $\mathrm{AD}$ skin lesions. Itching and how patients felt about their overall life quality were also analysed (which included items such as sleep, pain, ability to work or do normal leisure activities, etc.). The researchers found that if a patient had taken an NSISS for AD before taking dupilumab, it had no impact on the efficacy of dupilumab. All of the measurements evaluated improved significantly more in patients treated with dupilumab than in patients taking a placebo (dummy) medication. The benefits of treatment occurred within a few weeks of starting dupilumab treatment and remained until the end of the longest study included in this analysis, 1 year.

Keywords: Atopic dermatitis; Immuno suppressants; Clinical trial; Dupilumab

\section{Key Summary Points}

Why carry out this study?

Despite a questionable or poor risk-benefit profile, systemic non-steroidal immunosuppressants (NSISS) are frequently prescribed to treat adults with moderate-to-severe atopic dermatitis (AD) refractory to topical therapy.

Substantial evidence from large, randomized, double-blind, placebocontrolled clinical trials supports the use of dupilumab to treat adults with moderate-to-severe AD, with a favourable long-term safety profile.

\section{What did the study ask?}

This study assessed the impact of prior use of NSISS on the efficacy of dupilumab treatment.

\section{What were the study outcomes/conclusions?}

Regardless of prior systemic NSISS use, dupilumab provides rapid, significant, and sustained improvements in signs, symptoms, and quality of life in adult patients with moderate-to-severe $\mathrm{AD}$.

\section{What has been learned from the study?}

Overall, these results indicate that adult patients with $\mathrm{AD}$ and a history of systemic NSISS use do not have a reduced response to dupilumab treatment, supporting its use both as a systemic first-line treatment and in patients where systemic NSISS have failed.

\section{DIGITAL FEATURES}

This article is published with digital features, including a video, graphical abstract, to facilitate understanding of the article. To view digital 
features for this article go to https://doi.org/10. 6084/m9.figshare.14627706.

\section{INTRODUCTION}

Atopic dermatitis (AD) is a chronic, relapsing, type 2 inflammation skin disease characterized by skin lesions and pruritus that can significantly impair quality of life [1]. The disease affects up to $20 \%$ of children and $2-8 \%$ of adults worldwide [2, 3]. Type 2 immunity evolved to promote barrier immunity on mucosal surfaces and eliminate helminth parasites, and is characterized by Th2 $\mathrm{CD}^{+}{ }^{+} \mathrm{T}$ cells, IL-C 2 cells, and B cell production of IgE antibody subclass, recruitment of eosinophils, basophils, and mast cells, and release of signature cytokines that include interleukin (IL)-4, IL-5, IL-9, and IL-13 $[4,5]$.

The chronic nature of $\mathrm{AD}$ implies the need for long-term management, and topical treatments often provide inadequate control of moderate-to-severe AD [6-8]. Oral corticosteroids (OCS) are unsuitable for chronic or relapsing $\mathrm{AD}$ because of high likelihood of disease rebound [9]. Systemic non-steroidal immunosuppressants (NSISS), including cyclosporin A (CsA), methotrexate (MTX), mycophenolate mofetil, and azathioprine, are frequently prescribed to treat severe $\mathrm{AD}$ refractory to topical therapy (EUROSTAD [10]; TREAT $[11,12])$; however, there is a lack of robust evidence from large, well-designed randomized clinical trials (RCT) to support their use, and their toxicity profile requires frequent laboratory monitoring, and long-term treatment is not recommended because of a poor benefit-risk profile [13-18]. Patients treated with these broad-spectrum NSISS can suffer relapses and substantial side effects, including nephrotoxicity, liver dysfunction, and an increased risk of infection and cancer, and they are contraindicated in many patients $[13,15-22]$.

Dupilumab, a fully human $[23,24]$ monoclonal antibody to the IL-4R $\alpha$, blocks the shared receptor component for IL-4 and IL-13, key and central drivers in type 2 inflammatory diseases [25]. Dupilumab is approved in several countries for adolescents and adults with type 2 inflammatory diseases, including $\mathrm{AD}$ and asthma, and in adults with chronic rhinosinusitis with nasal polyps. It is also approved in the USA for children $6-11$ years with moderateto-severe AD, and in Europe and other countries for children 6-11 years with severe AD. The dupilumab development programme included more than 4000 patients with moderate-to severe AD globally, and more than 10,000 patients in all indications..

In dupilumab $\mathrm{AD}$ clinical trials, a large proportion of patients had prior treatment with at least one systemic NSISS agent. Here we analyse the efficacy of dupilumab vs. control in these patients, using clinically meaningful endpoints recommended by a global consensus of dermatologists [26], comparing the results achieved in dupilumab-treated vs. placebo/control-treated patients with prior use of NSISS and patients naïve to NSISS use.

The objective of this study was to assess dupilumab treatment effect in patients with moderate-to-severe $\mathrm{AD}$, comparing patients with and without prior systemic NSISS use vs. control groups.

\section{METHODS}

\section{Study Design}

This post hoc analysis includes data from four randomized, double-blind, placebo-controlled, parallel-group, international phase 3 clinical trials: LIBERTY AD SOLO 1 (NCT02277743) and LIBERTY AD SOLO 2 (NCT02277769), for which data have been pooled [27]; LIBERTY AD CAFÉ (NCT02755649) [28]; and LIBERTY AD CHRONOS (NCT02260986) [29]. Detailed methodology, primary efficacy, and safety results have been reported previously [27-29].

SOLO 1 and SOLO 2 were two identically designed phase 3 trials that evaluated the efficacy and safety of dupilumab monotherapy treatment (300 mg weekly [qw] or every 2 weeks [q2w]) for 16 weeks in adult patients with moderate-to-severe AD. A 35-day screening and washout period preceded study drug administration. The CAFÉ and CHRONOS studies evaluated dupilumab treatment (300 mg qw or q2w) 
with concomitant topical corticosteroids (TCS) in adults with moderate-to-severe $\mathrm{AD}$ for 16 and 52 weeks, respectively. A 35-day screening and washout period for systemic treatments preceded study drug administration, TCS were allowed during this period. CAFÉ included only adult patients with $\mathrm{AD}$ and an inadequate response or intolerance to CsA or for whom this treatment was medically inadvisable.

Baseline data for patient demographics, characteristics, and prior use of NSISS from all four clinical trials were very similar, and thus pooled. Efficacy data at 16 weeks from CAFÉ and CHRONOS were pooled to assess dupilumab treatment with concomitant TCS. Of dupilumab-treated patients, all analyses included only patients randomized to the approved dupilumab dosing regimen of $300 \mathrm{mg}$ q2w $[30,31]$. Patients have been stratified by at least one prior or no prior use of systemic NSISS. Around $10-11 \%$ of patients with previous treatment with NSISS received at least two NSISS (Table S1 in the supplementary material).

These trials were approved by respective institutional review boards and conducted in accordance with the ethical principles outlined in the Declaration of Helsinki, the International Conference on Harmonization Good Clinical Practice guidelines, and applicable regulatory requirements. All patients provided written informed consent before participating in the trial.

This analysis includes results for change from baseline in Eczema Area and Severity Index (EASI; range 0-72), SCORing AD (SCORAD; range 0-103), Dermatology Life Quality Index (DLQI; range 0-30), Patient-Oriented Eczema Measure (POEM; range 0-28); percentage of patients achieving: $\geq 3$-point improvement in Peak Pruritus Numerical Rating Scale (NRS), $\geq 75 \%$ improvement from baseline in EASI (EASI-75), EASI $\leq 7$, Peak Pruritus NRS $\leq 4$, POEM $\leq 7$, DLQI $\leq 5$; analysis of patients overall well-being related to the disease (PGADS) and patient perception of treatment effect (PGATE) on a 5-point Likert $t$ scale (poor, fair, good, very good, and excellent).

\section{Statistical Analysis}

Efficacy analyses were performed separately in each subgroup (patients with or without prior systemic NSISS use) on the full analysis set, which included all randomized patients. For continuous outcomes, patients missing an assessment or who received rescue treatment were censored and set to missing and then imputed using the multiple imputation method; $p$ values were assessed using an analysis of covariance model at each visit with baseline measurement as a covariate and the treatment, region, baseline Investigator's Global Assessment (IGA) strata $(\mathrm{IGA}=3$ vs. $\mathrm{IGA}=4)$ and study identifier (for the SOLO studies only) as fixed factors. For responder endpoints, patients missing an assessment or who received rescue treatment were censored and set to missing; $p$ values were derived by Cochran-Mantel-Haenszel (CMH) test stratified by baseline disease severity and study identifier at each visit. For PGADS and PGATE, patients missing an assessment or who received rescue treatment were set to worst class (poor). Means were calculated using the least-squares (LS) method. As all analyses were conducted post hoc, all $p$ values should be considered to be nominal. Analyses were performed using SAS V9.4 or higher.

\section{RESULTS}

\section{Patients}

A total of 1553 patients randomized to placebo/control or dupilumab treatment were included in this analysis, then stratified by prior or no prior systemic NSISS use. Baseline demographics were very similar between patients with a history of prior NSISS treatment and those naïve to such treatment (Table 1). Patients who had prior use of systemic NSISS presented with, on average, numerically slightly higher disease severity at baseline, except for itch (Peak Pruritus NRS), which was similar (Table 1). 
Table 1 Baseline demographics, disease characteristics and prior systemic non-steroidal immunosuppressants (NSISS) use. Baseline data is pooled from patients from the individual studies: SOLO1, SOLO2, CAFÉ, and CHRONOS

\section{Baseline demographics and disease characteristics}

\begin{tabular}{|c|c|c|c|c|}
\hline & \multicolumn{2}{|c|}{0 prior systemic NSISS } & \multicolumn{2}{|c|}{$\geq 1$ prior systemic NSISS } \\
\hline & $\begin{array}{l}\text { Control } \\
(N=606)\end{array}$ & $\begin{array}{c}\text { Dupilumab } \\
300 \mathrm{mg} \text { q2w } \\
(N=444)\end{array}$ & $\begin{array}{l}\text { Control } \\
(N=277)\end{array}$ & $\begin{array}{c}\text { Dupilumab } \\
300 \mathrm{mg} \mathrm{q} 2 \mathrm{w} \\
(\boldsymbol{N}=226)\end{array}$ \\
\hline Age, mean (SD), years & $37.4(13.7)$ & $38.0(14.4)$ & $38.7(13.4)$ & $39.3(13.3)$ \\
\hline Sex, male, $n(\%)$ & $332(54.8)$ & $253(57.0)$ & $179(64.6)$ & $141(62.4)$ \\
\hline \multicolumn{5}{|l|}{ Race, $n(\%)$} \\
\hline White & $407(67.2)$ & $326(73.4)$ & $207(74.7)$ & $172(76.1)$ \\
\hline Black/African American & $49(8.1)$ & $20(4.5)$ & $6(2.2)$ & $5(2.2)$ \\
\hline Asian & $133(21.9)$ & $85(19.1)$ & $58(20.9)$ & $44(19.5)$ \\
\hline Other & $17(2.8)$ & $13(2.9)$ & $6(2.2)$ & $5(2.2)$ \\
\hline Weight, kg, mean (SD) & $76.0(18.8)$ & $76.5(18.0)$ & $76.1(17.5)$ & $74.6(17.4)$ \\
\hline Duration of $\mathrm{AD}$, years, mean $(\mathrm{SD})$ & $28.1(14.2)$ & $27.7(15.2)$ & $29.1(15.0)$ & $30.0(15.6)$ \\
\hline EASI $(0-72)$, mean $(S D)$ & $32.9(13.6)$ & $31.5(12.6)$ & $34.3(13.1)$ & $35.0(13.0)$ \\
\hline Patients with IGA score $4, n(\%)$ & $275(45.4)$ & $188(42.3)$ & $149(53.8)$ & $138(61.1)$ \\
\hline $\begin{array}{l}\text { Peak Pruritus NRS }(0-10) \text {, mean } \\
\text { (SD) }\end{array}$ & $7.2(1.9)$ & $7.3(1.9)$ & $7.4(1.9)$ & $7.3(1.7)$ \\
\hline $\begin{array}{l}\text { SCORAD total score (103), mean } \\
\text { (SD) }\end{array}$ & $67.0(13.9)$ & $66.2(13.6)$ & $68.8(13.9)$ & $70.5(13.4)$ \\
\hline POEM $(0-28)$, mean $(S D)$ & $19.8(5.9)$ & $19.6(6.2)$ & $21.1(6.2)$ & $21.2(5.4)$ \\
\hline DLQI $(0-30)$, mean $(S D)$ & $14.2(7.5)$ & $14.2(7.3)$ & $15.8(7.3)$ & $15.4(7.3)$ \\
\hline
\end{tabular}

Distribution of patients with $\geq 1$ prior use of systemic NSISS

\begin{tabular}{lll}
\hline & Control, $\boldsymbol{n}(\%)$ & Dupilumab 300 $\mathbf{~ m g ~ q 2 w , ~} \boldsymbol{n}(\%)$ \\
\hline Pts with $\geq 1$ prior use of ISS & $277(100.0)$ & $226(100.0)$ \\
Cyclosporin & $226(81.6)$ & $186(82.3)$ \\
Methotrexate & $72(26.0)$ & $51(22.6)$ \\
Azathioprine & $48(17.3)$ & $34(15.0)$ \\
Mycophenolate mofetil & $30(10.8)$ & $34(15.0)$
\end{tabular}

$A D$ atopic dermatitis, EASI Eczema Area and Severity Index, DLQI Dermatology Life Quality Index, IGA Investigator's Global Assessment, NSISS non-steroidal immunosuppressants, NRS numerical rating scale, POEM Patient-Oriented Eczema Measure, $q 2 w$ every 2 weeks, SCORAD SCORing Atopic Dermatitis, $S D$ standard deviation 
A

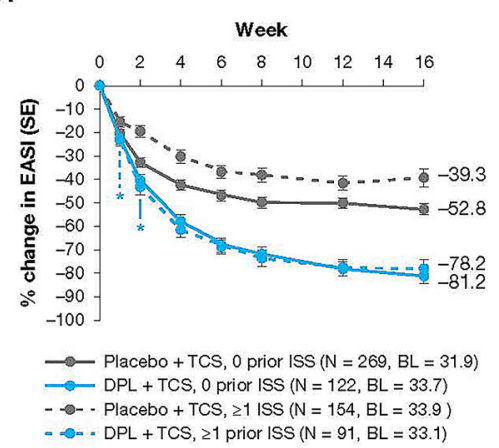

D

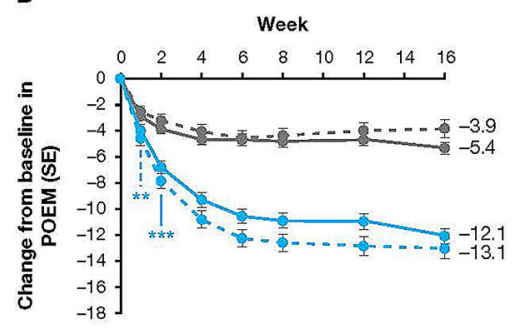

$\Longrightarrow$ Placebo + TCS, 0 prior ISS $(\mathrm{N}=269, \mathrm{BL}=19.3)$ $\longrightarrow$ DPL + TCS, 0 prior ISS $(\mathrm{N}=122, \mathrm{BL}=18.9$ ) - - Placebo + TCS, $\geq 1$ prior ISS $(\mathrm{N}=154, \mathrm{BL}=20.6)$ - - DPL + TCS, 21 prior ISS $(\mathrm{N}=91, \mathrm{BL}=21.0)$

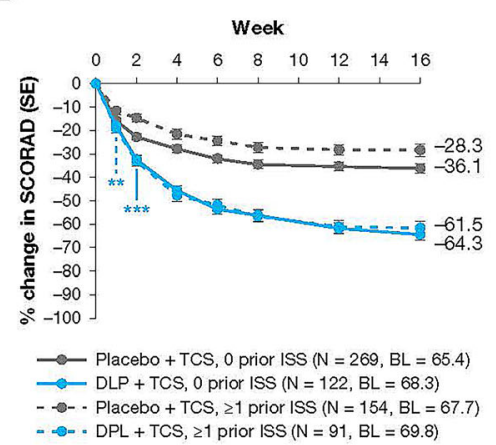

C

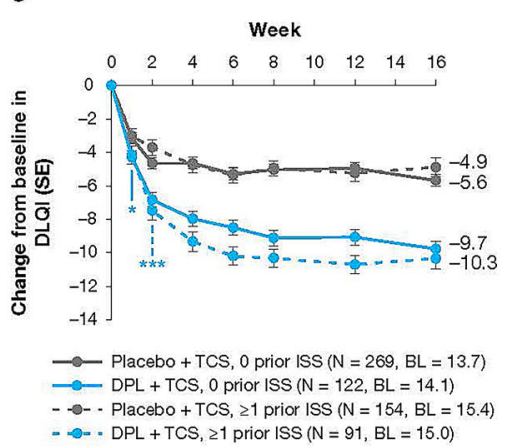

E

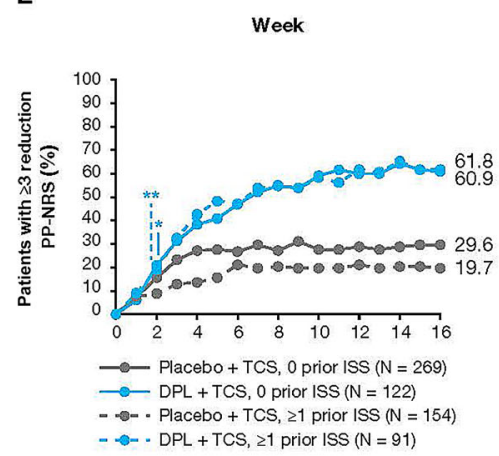

Fig. 1 Efficacy of short-term (16 weeks) dupilumab $300 \mathrm{mg} \mathrm{q} 2 \mathrm{w}$ with concomitant TCS therapy for patients with atopic dermatitis with or without prior use of nonsteroidal immunosuppressants (pooled analysis from CAFÉ and CHRONOS trials). a LS mean percentage change from baseline in EASI. b LS mean percentage change from baseline in SCORAD. c LS mean change from baseline in DLQI. $\mathbf{d}$ LS mean change from baseline in POEM. e Percentage of patients achieving $\geq 3$-point improvement in Peak Pruritus NRS from baseline. ${ }^{*} p<0.05$ vs. placebo; ${ }^{* *} p<0.01$ vs. placebo;

\section{Dupilumab Efficacy in Patients With or Without Prior Systemic NSISS Use}

All dupilumab-treated patient groups in this analysis, regardless of concomitant TCS use or prior use of systemic NSISS, when compared vs. control, achieved a significantly higher percentage reduction from baseline in EASI by week 4; and SCORAD, DLQI, and POEM by week 2; (Figs. 1, 2, and 3); $\geq 3$-point improvement in Peak Pruritus NRS by week 2 , and 4 point improvement by week 3 ; DLQI score $\leq 5$ by week 2; and EASI score $\leq 7$ and POEM score $\leq 7$ by week 4 (Figs. 1, 2, and 3).
${ }^{* * *} p<0.001$ vs. placebo. AD atopic dermatitis, BL baseline, EASI Eczema Area and Severity Index, DPL dupilumab, DLQI Dermatology Life Quality Index, ISS immunosuppressant, NSISS non-steroidal immunosuppressants, POEM Patient-Oriented Eczema Measure, PPNRS Peak Pruritus NRS, q2w every 2 weeks, SCORAD SCORing Atopic Dermatitis, SE standard error, TCS topical corticosteroids

By week 16 of dupilumab with concomitant TCS treatment (pooled analysis of CAFÉ and CHRONOS trials), patients in both populations achieved significant $(p<0.001)$ improvements in EASI, SCORAD, DLQI, POEM, and Peak Pruritus NRS.

Further improvements in these outcome measures were seen following long-term treatment of dupilumab with concomitant TCS (52 weeks, CHRONOS). Patients achieved an LS mean percentage reduction in EASI from baseline (vs. placebo with TCS) of $-89.0 \%$ vs. $-66.6 \%$, 95\% LSMCI $(-32.20,-12.45)$ and $-84.1 \% \quad$ vs. $-55.6 \%, \quad 95 \% \quad$ LSMCI 
A
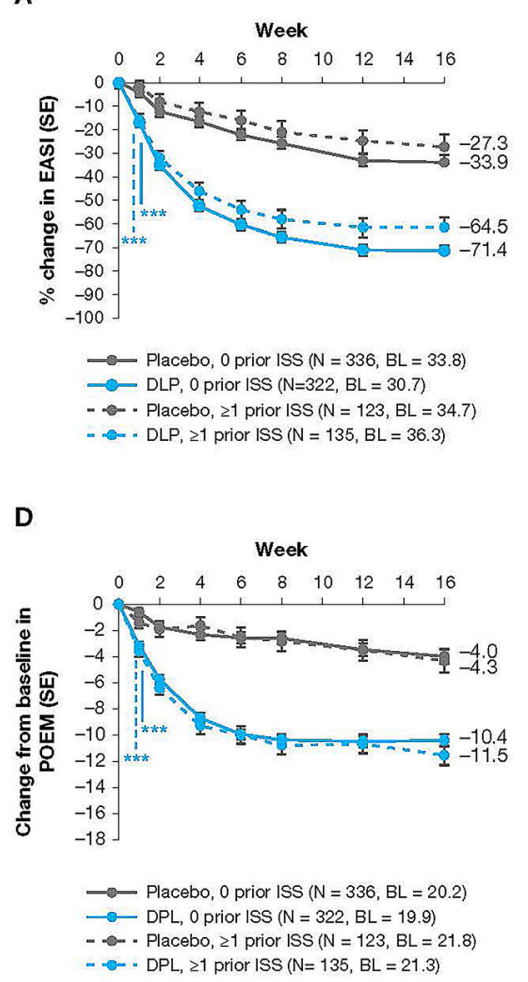

B

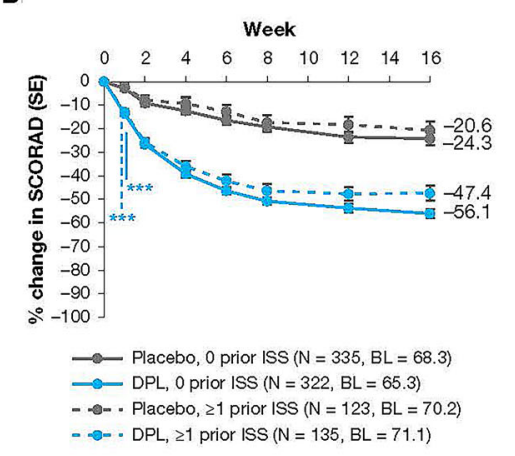

E

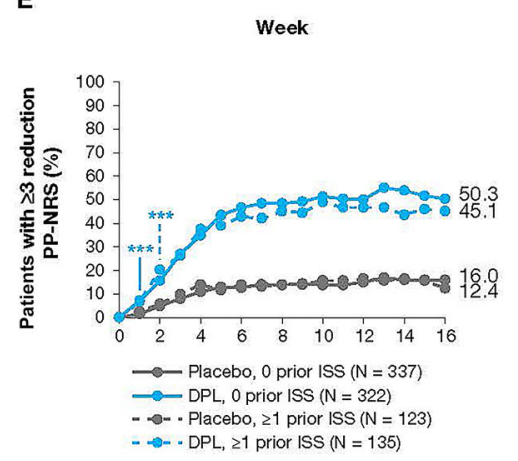

c

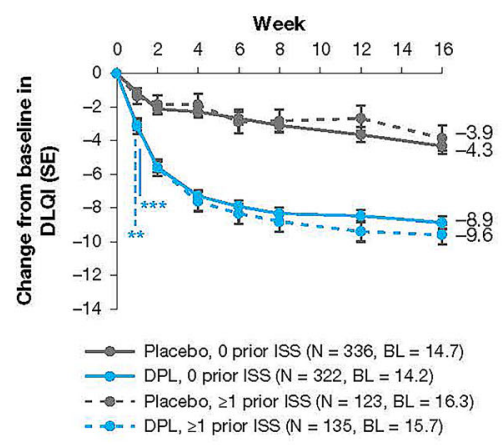

Fig. 2 Efficacy of dupilumab $300 \mathrm{mg}$ q2w monotherapy for atopic dermatitis patients with or without prior use of non-steroidal immunosuppressants (SOLO $1 \&$ SOLO 2). a LS mean percentage change from baseline in EASI. b LS mean percentage change from baseline in SCORAD. $\mathbf{c}$ LS mean change from baseline in DLQI. $\mathbf{d}$ LS mean change from baseline in POEM. e Percentage of patients achieving $\geq 3$-point improvement in Peak Pruritus NRS from baseline. ${ }^{*} p<0.05$ vs. placebo; ${ }^{* *} p<0.01$ vs. placebo; ${ }^{* * *} p<0.001$ vs. placebo. AD atopic dermatitis, BL

$(-47.28,-9.68) ;-73.0 \%$ vs. $-49.6 \%, 95 \%$ LSMCI $(-32.54,-14.24) ;$ and $-67.5 \%$ vs. $-43.5 \%$ 95\% LSMCI $(-37.81,-10.04)$ in SCORAD; and an LS mean reduction of -10.2 vs. $-6.8, \quad 95 \% \quad$ LSMCI $\quad(-5.002,-1.888)$ and $-12.2 \quad$ vs. $-7.2, \quad 95 \% \quad$ LSMCI $(-7.354,-2.548)$ in DLQI; -12.7 vs. -6.1 , 95\% LSMCI $(-8.918,-4.278)$ and -15.8 vs. $-5.1,95 \%$ LSMCI $(-14.029,-7.365)$ in POEM (Fig. 2d); and $56.5 \%$ vs. $19.0 \%, 95 \%$ CI risk difference (23.97-50.94) and $48.8 \%$ vs. $11.3 \%$, 95\% CI risk difference (21.40-53.63) achieved $\geq 3$-point improvement in Peak Pruritus NRS, in the NSISS and the at least one prior baseline, EASI Eczema Area and Severity Index, DPL dupilumab, DLQI Dermatology Life Quality Index, ISS immunosuppressant, NSISS non-steroidal immunosuppressants, POEM Patient-Oriented Eczema Measure, PPNRS Peak Pruritus NRS, q2w every 2 weeks, SCORAD SCORing Atopic Dermatitis, SE standard error, TCS topical corticosteroids

use of a systemic NSISS populations, respectively.

In both the short- and long-term studies, following treatment with placebo plus concomitant TCS, patients with at least one prior use of a systemic NSISS achieved consistent lower improvements in signs (EASI), SCORAD and itch (Peak Pruritus NRS) than patients naïve to systemic NSISS.

Significant improvements $(p<0.001)$ in outcome measures were also evident in patients after receiving 16 weeks of dupilumab monotherapy (pooled SOLO 1 and SOLO 2 trials) regardless of prior use of NSISS (Fig. 2). 

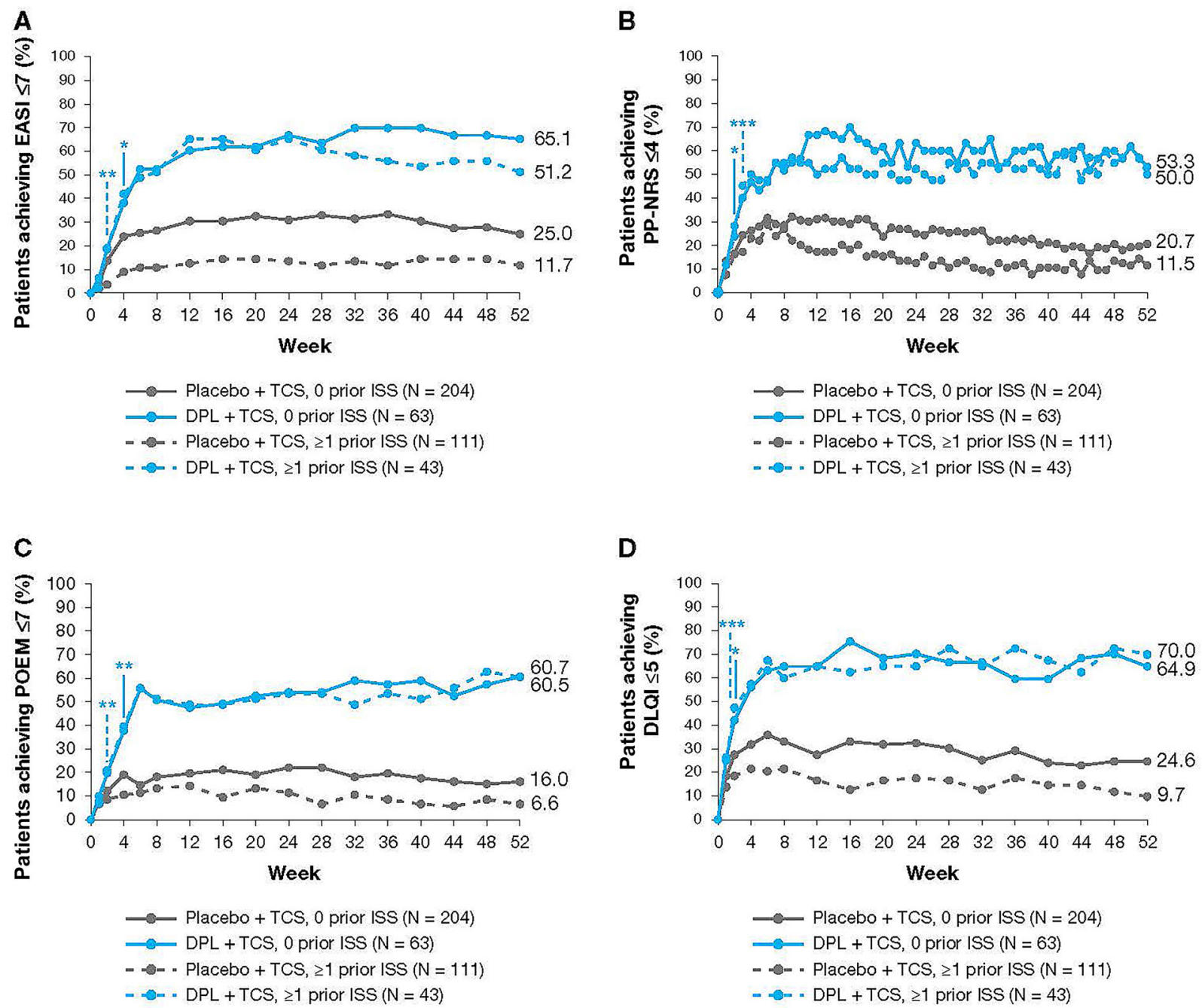

D

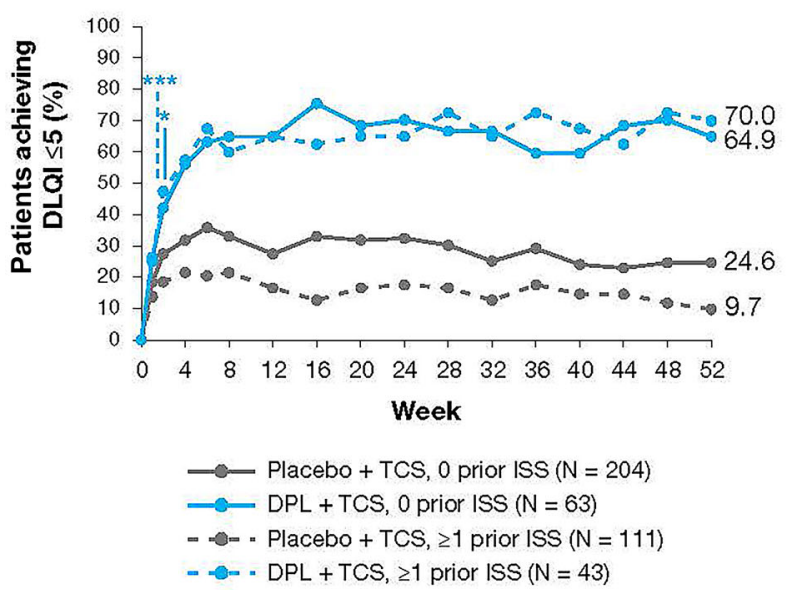

Fig. 3 Clinically meaningful responses following dupilumab $300 \mathrm{mg}$ q2 $\mathrm{w}$ with concomitant TCS therapy over 52 weeks (CHRONOS) with or without prior use of nonsteroidal immunosuppressants. a Percentage of patients achieving EASI score of $\leq 7$. b Percentage of patients achieving Peak Pruritus NRS of $\leq 4$. c Percentage of Patients achieving POEM score of $\leq 7$. $\mathbf{d}$ Percentage of Patients achieving DLQI score of $\leq 5 .{ }^{*} p<0.05$ vs. placebo; ${ }^{* *} p<0.01$ vs. placebo; ${ }^{* * *} p<0.001$ vs. placebo.

In a set of clinically meaningful response analyses (Fig. 3), by week 8 the majority of patients receiving dupilumab and TCS (CHRONOS), with or without prior systemic NSISS treatment, achieved scores corresponding to minimal/mild or absent disease: EASI score $\leq 7$, Peak Pruritus NRS $\leq 4$, POEM score $\leq 7$, and DLQI score $\leq 5$. These improvements were

$\mathrm{AD}$ atopic dermatitis, BL baseline, EASI Eczema Area and Severity Index, DPL dupilumab, DLQI Dermatology Life Quality Index, ISS immunosuppressant, NSISS nonsteroidal immunosuppressants, POEM Patient-Oriented Eczema Measure, PP-NRS Peak Pruritus NRS, q2w every 2 weeks, TCS topical corticosteroids

maintained until the end of treatment (52 weeks). In addition, by week 2 , over $60 \%$ of patients receiving dupilumab with concomitant TCS, regardless of their prior use of NSISS, rated their overall well-being in relation to their skin condition as "good", "very good", or "excellent" (Fig. 4). For patients receiving placebo and TCS only, numerically fewer patients with a history 

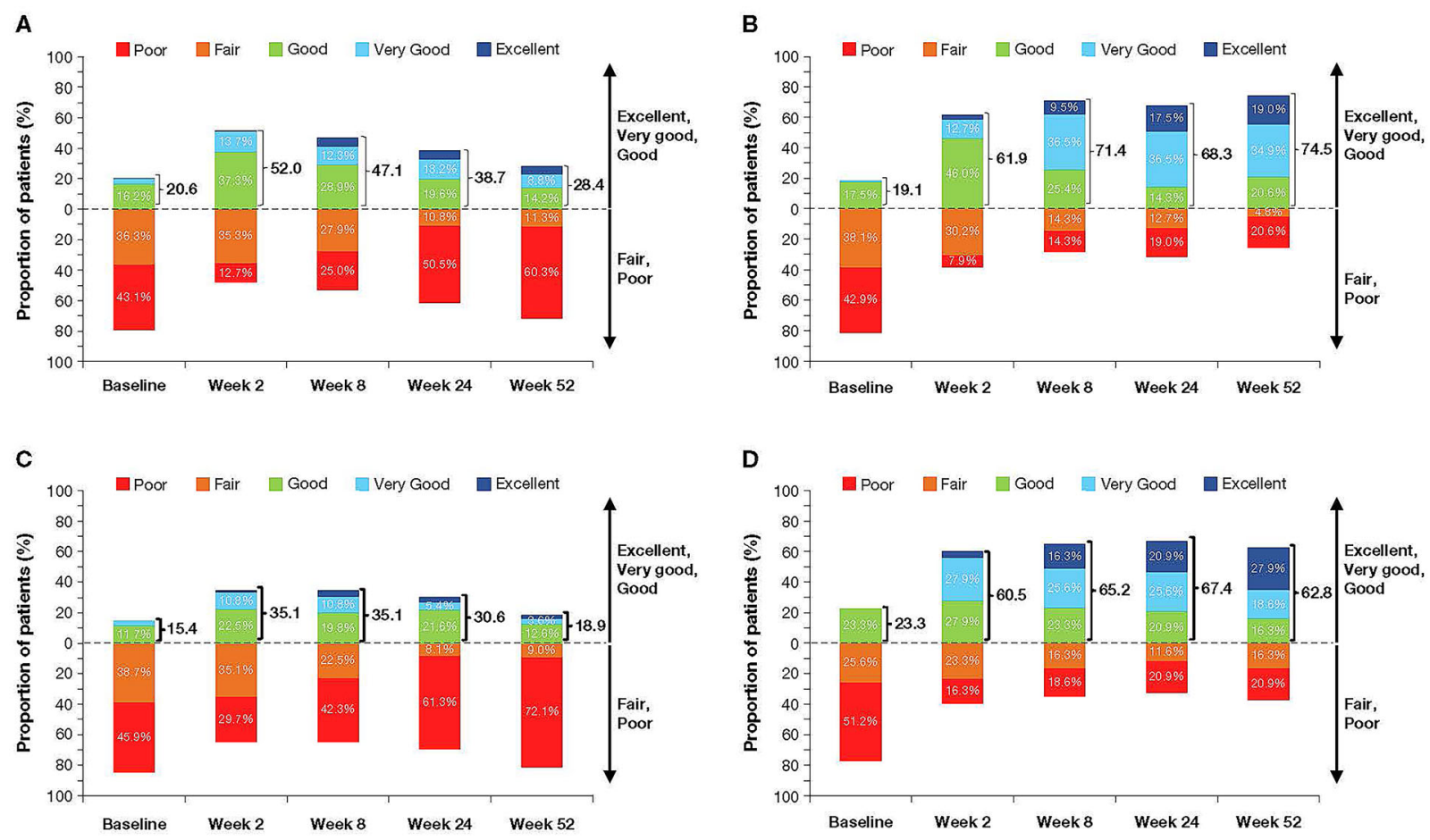

Fig. 4 Patient Global Assessment of Disease Status following dupilumab $300 \mathrm{mg} \mathrm{q2w}$ with concomitant TCS therapy over 52 weeks (CHRONOS). Patients were asked: "Considering all the ways in which your eczema affects you, indicate how well you are doing” and rated on a 5-point scale. a Patients with 0 prior systemic NSISS use receiving placebo and TCS. b Patients with 0 prior systemic NSISS use receiving dupilumab and TCS.

of prior systemic NSISS use achieved these clinically meaningful responses, compared with patients naïve to NSISS.

During dupilumab with concomitant TCS treatment (CHRONOS 52 weeks), over half of patients in both populations (those with or without prior use of systemic NSISS) achieved EASI-75 (by week 6, data not shown), and more than $3 / 4$ rated their satisfaction with treatment as "good", "very good", or "excellent" (by week through to end of treatment, data not shown).

\section{DISCUSSION}

In this analysis, dupilumab therapy (with or without concomitant TCS therapy) resulted in rapid, consistent, and significant improvements

in signs and symptoms of $\mathrm{AD}$, as well as improvements in quality of life (QoL) compared to control arms (placebo/placebo + TCS). Improvements were achieved by patients naïve to systemic NSISS, and also by patients with a history of use of these agents. In both patient populations, these improvements were sustained throughout 1 year (end of treatment).

A majority of patients treated with dupilumab (with or without prior NSISS use) achieved clinically meaningful responses corresponding to absent or mild/minimal disease signs and symptoms, and low or no impact in QoL by the end of the treatment period. Most patients considered their general well-being in relation to their $\mathrm{AD}$ as good, very good, or excellent, and were very satisfied with the treatment effect. 
Of note, as observed in the control groups, patients with prior systemic NSISS use were consistently less responsive to TCS treatment without dupilumab. These patients had poorer improvements in signs and symptoms compared to the NSISS-naïve patients. These data suggest that patients may become less responsive to TCS treatment following prior use of systemic NSISS, or that these patients constitute a more "difficult to treat with TCS" population, and thus had previously been recommended systemic therapy. Interestingly, however, patients with prior use of NSISS were generally not more "difficult to treat" with dupilumab, compared with those naïve to systemic NSISS.

$\mathrm{AD}$ can have a profound impact on a patient's QoL from direct and indirect effects of signs and symptoms $[32,33]$. Even with slightly higher baseline disease severity in terms of subjective QoL impairment (DLQI) and patientreported symptoms (POEM), numerically higher improvements in DLQI and POEM were observed in patients with a history of systemic NSISS use compared with those patients naïve to these agents, suggesting a greater perceived relief. Relief was also evident in pruritus. Comparable baseline severity in Peak Pruritus NRS between patients with or without prior use of systemic NSISS was matched with comparable improvements following dupilumab treatment, with the majority of patients achieving absent or mild pruritus at end of treatment, independent of prior treatment with NSISS.

The similar baseline disease severity between patients with or without prior use of systemic NSISS is likely a consequence of clinical trial entry criteria. In dupilumab phase 3 trials, the AD severity of patients at baseline was similar to that of patients who are candidates for systemic therapy in EU registries (BioDay [34]; EUROSTAD [10]; TREAT [12]). In a retrospective Korean study, baseline severity of disease was higher in NSISS-experienced patients (EASI 31.7 in the prior NSISS group vs. 26.9 in the NSISSnaïve group) [35]. Therefore, patients in this analysis are likely representative of patients with $\mathrm{AD}$ who are candidates for systemic treatment in the real-world population, many of whom will have had prior NSISS therapy.
In the absence of a head-to-head study comparing dupilumab vs. any systemic NSISS, an indirect comparison of dupilumab and CsA published by Ariëns et al. in 2019 [36] suggests a higher relative efficacy of dupilumab vs. CsA. The study found that $74 \%$ of patients treated with dupilumab achieved EASI-75 at 24-30 weeks, vs. only $40 \%$ of those treated with CsA. In addition, registry studies show higher adherence to dupilumab treatment, compared to CsA and methotrexate [34, 37]. Ongoing registry studies (EUROSTAD [10]; TREAT [12]) are further investigating long-term response and adherence to systemic treatments.

The results from these analyses involving 1553 patients from four robust RCTs are supported by real-world cases of dupilumab treatment [38], where dupilumab has provided better short- and long-term control of a patient's AD compared with systemic NSISS $[37,39]$ and has been similarly effective in patients who have failed to respond to CsA $[37,39,40]$ and even in a cohort of patients who failed treatment on two or more NSISS [34].

Limitations of the study include the post hoc nature of the analyses.

\section{CONCLUSIONS}

Taken together, these results indicate that a patient's prior history of use of systemic NSISS does not impact the efficacy of dupilumab treatment for moderate-to-severe $\mathrm{AD}$, supporting its use both as a systemic first-line treatment and in patients in whom systemic NSISS have been used. Dupilumab brings a new perspective to systemic treatment of this patient population, offering the prospect of long-term control [41] and flare prevention [42].

\section{ACKNOWLEDGEMENTS}

We thank patients and investigators who participated in the studies, El-Bdaoui Haddad, Linda Williams, and Adriana Mello. 
Author Contributions. Ana B Rossi: conceptualization, data analysis definition and interpretation, coordination, co-writing original draft with medical writer, review \& editing of following drafts, and approval of final manuscript; Sonya Cyr: conceptualization, data analysis definition and interpretation, review \& editing of drafts, and approval of final manuscript; Jérôme Msihid, Zhen Chen: data analysis, methodology, review \& editing of drafts and approval of final manuscript; Christopher Griffiths, Marjolein de Bruin-Weller, Mette Deleuran, Maria Concetta Fargnoli, Delphine Staumont-Sallé, Chih-ho-Hong, Jose SánchezCarazo, Peter Foley, Seong Jun Seo: investigator in at least one of the studies included in the analyses, review \& editing of all drafts, and final manuscript.

Funding. Research sponsored by Sanofi and Regeneron Pharmaceuticals, Inc. The journal's rapid service fee was funded by Sanofi and Regeneron Pharmaceuticals Inc. Funded in part by the National Institute for Health Research (NIHR) Manchester Biomedical Research Centre.

Medical Writing and Editorial Assistance. Medical writing and editorial support was provided by Jamie Casswell, PhD, and Carolyn Ellenberger, PhD, of Excerpta Medica, and was funded by Sanofi Genzyme and Regeneron Pharmaceuticals, Inc., according to the Good Publication Practice guideline.

Authorship. All named authors meet the International Committee of Medical Journal Editors (ICMJE) criteria for authorship for this manuscript, take responsibility for the integrity of the work as a whole, and have given final approval for the version to be published.

Disclosures. Christopher Griffiths has received honoraria and/or research grants from AbbVie, Almirall, Eli Lilly, Janssen, LEO Pharma, Merck, Novartis, Pfizer, Regeneron Pharmaceuticals, Inc., Roche, Sandoz, Sanofi, Sun Pharma, UCB. Marjolein de Bruin-Weller has been Principal Investigator, advisory board member, consultant for Regeneron
Pharmaceuticals, Inc., Sanofi Genzyme; Principal Investigator, advisory board member for AbbVie, LEO Pharma, Pfizer; advisory board member for Eli Lilly, Galderma, Janssen, UCB. Mette Deleuran has received research support, has consulting/advisory board agreements, and/ or has received honoraria for lectures from AbbVie, Almirall, Eli Lilly, LEO Pharma, Pfizer, Pierre Fabre, Regeneron Pharmaceuticals, Inc., Sanofi Genzyme. Maria Concetta Fargnoli: Abbvie, Almirall, Bristol Myers Squibb, Eli Lilly, Galderma, Janssen, LEO Pharma, MSD, Medac Pharma, Mylan, Novartis, Pfizer, Pierre Fabre, Sanofi Genzyme, Roche, Sun Pharma, UCB. Delphine Staumont-Sallé has been speaker, investigator, consultant for Abbvie, AstraZeneca, Eli Lilly, Janssen, LEO Pharma, Novartis, Pfizer, Regeneron Pharmaceuticals, Inc., Sanofi Genzyme. Chih-ho Hong has been investigator for Arcutis, Antiobix, Centocor, Cutanea, MedImmune; consultant for sanofi-aventis; investigator, consultant for Boehringer Ingelheim, GlaxoSmithKline; speaker for Cipher Pharmaceuticals; speaker, investigator, consultant for AbbVie, Amgen, Bausch Health, Bristol Myers Squibb, Dermavant, Eli Lilly, Galderma, Janssen, LEO Pharma, Novartis, Regeneron Pharmaceuticals, Inc., Roche, Sun Pharma, UCB. Jose Sánchez-Carazo has been Principal Investigator, advisory board member, and received honoraria and/or research grants from AbbVie, Almirall, Amgen, Eli Lilly, Janssen, LEO Pharma, Novartis, Pfizer, Roche, Sandoz, Sanofi, Sun Pharma, UCB. Peter Foley served as advisor and/or consultant and/or received speaker's bureau/honoraria and/or received research and/ or travel grants and/or participated in clinical trials (investigator) for AbbVie, Amgen, Arcutis Antiobix, Aslan Pharmaceuticals, AstraZeneca, Bristol Myers Squibb, Boehringer Ingelheim, Botanix Pharmaceuticals, Celtaxsys, CSL, Cutanea, Dermira, Eli Lilly, Galderma, Geneseq Biosciences, Genentech, GlaxoSmithKline, Hexima, Janssen, LEO Pharma, Mayne Pharma, MedImmune, Merck, Novartis, Pfizer, Regeneron Pharmaceuticals Inc, Reistone Biopharma, Roche, Sanofi, Sun Pharma, UCB Pharma, Valeant, Wintermute Biomedical. Seong Jun Seo has been speaker, investigator, consultant for Abbvie, Eli Lilly, LEO Pharma, Sanofi Genzyme. 
Jérôme Msihid is an employee, may hold stock and/or stock options in Sanofi. Zhen Chen, Sonya L. Cyr are employees and shareholders of Regeneron Pharmaceuticals, Inc. Ana B. Rossi is an employee, may hold stock and/or stock options in Sanofi Genzyme.

Compliance with Ethics Guidelines. All trials were approved by respective institutional review boards and conducted in accordance with the ethical principles outlined in the Declaration of Helsinki, the International Conference on Harmonization Good Clinical Practice guidelines, and applicable regulatory requirements. All patients or carers provided written informed consent before participating in the trial. The studies presented in this paper have been approved by their respective institutional review boards which are included in this submission as supplementary material.

Data Availability. The datasets generated during and/or analyzed during the current study are available from the corresponding author on reasonable request.

Open Access. This article is licensed under a Creative Commons Attribution-NonCommercial 4.0 International License, which permits any non-commercial use, sharing, adaptation, distribution and reproduction in any medium or format, as long as you give appropriate credit to the original author(s) and the source, provide a link to the Creative Commons licence, and indicate if changes were made. The images or other third party material in this article are included in the article's Creative Commons licence, unless indicated otherwise in a credit line to the material. If material is not included in the article's Creative Commons licence and your intended use is not permitted by statutory regulation or exceeds the permitted use, you will need to obtain permission directly from the copyright holder. To view a copy of this licence, visit http://creativecommons.org/licenses/bync/4.0/.

\section{REFERENCES}

1. Weidinger S, Novak N. Atopic dermatitis. Lancet. 2016;387(10023):1109-22.

2. Silverberg JI, Barbarot S, Gadkari A, et al. Atopic dermatitis in the pediatric population: a cross-sectional, international, epidemiologic study. Ann Allergy Asthma Immunol. 2021;126(4):417-428.e2.

3. Barbarot S, Auziere S, Gadkari A, et al. Epidemiology of atopic dermatitis in adults: results from an international survey. Allergy. 2018;73(6):1284-93.

4. Gandhi NA, Bennett BL, Graham NM, Pirozzi P, Stahl N, Yancopoulos GD. Targeting key proximal drivers of type 2 inflammation in disease. Nat Rev Drug Discov. 2016;15(1):35-50.

5. LePoidevin LM, Lee DE, Shi VY. A comparison of international management guidelines for atopic dermatitis. Pediatr Dermatol. 2019;36(1):36-65.

6. Furue $\mathrm{M}$, Terao $\mathrm{H}$, Moroi $\mathrm{Y}$, et al. Dosage and adverse effects of topical tacrolimus and steroids in daily management of atopic dermatitis. J Dermatol. 2004;31(4):277-83.

7. Furue $\mathrm{M}$, Terao $\mathrm{H}$, Rikihisa $\mathrm{W}$, et al. Clinical dose and adverse effects of topical steroids in daily management of atopic dermatitis. Br J Dermatol. 2003;148(1):128-33.

8. Haeck IM, Rouwen TJ, Timmer-de Mik L, de BruinWeller MS, Bruijnzeel-Koomen CA. Topical corticosteroids in atopic dermatitis and the risk of glaucoma and cataracts. J Am Acad Dermatol. 2011;64(2):275-81.

9. Leung DY, Boguniewicz M, Howell MD, Nomura I, Hamid QA. New insights into atopic dermatitis. J Clin Invest. 2004;113(5):651-7.

10. Gerbens LAA, Apfelbacher CJ, Irvine AD, et al. TREatment of ATopic eczema (TREAT) Registry Taskforce: an international Delphi exercise to identify a core set of domains and domain items for national atopic eczema photo- and systemic therapy registries. Br J Dermatol. 2019;180(4):790-801.

11. Vermeulen FM, Gerbens LAA, Schmitt J, et al. The European TREatment of ATopic eczema (TREAT) Registry Taskforce survey: prescribing practices in Europe for phototherapy and systemic therapy in adult patients with moderate-to-severe atopic eczema. Br J Dermatol. 2020;183(6):1073-82.

12. Alexander H, Patton T, Jabbar-Lopez ZK, Manca A, Flohr C. Novel systemic therapies in atopic dermatitis: what do we need to fulfil the promise of a treatment revolution? F1000Res. 2019;8(F1000 
Faculty Rev):132. https://doi.org/10.12688/ f1000research.17039.1.

13. Roekevisch E, Spuls PI, Kuester D, Limpens J, Schmitt J. Efficacy and safety of systemic treatments for moderate-to-severe atopic dermatitis: a systematic review. J Allergy Clin Immunol. 2014;133(2): 429-38.

14. de Bruin-Weller M, Pink AE, Patrizi A, et al. EUROSTAD prospective observational study: baseline characteristics, atopic dermatitis severity, and patient-reported outcomes. J Am Acad Dermatol. 2019;81:P-161.

15. Ryan C, Amor KT, Menter A. The use of cyclosporine in dermatology: part II. J Am Acad Dermatol. 2010;63(6):949-74.

16. Sidbury R, Davis DM, Cohen DE, et al. Guidelines of care for the management of atopic dermatitis: section 3. Management and treatment with phototherapy and systemic agents. J Am Acad Dermatol. 2014;71(2):327-49.

17. European Medicines Agency. Assessment report for Sandimmun Neoral and associated names. 2013. https://www.ema.europa.eu/en/documents/referral/ sandimmun-neoral-article-30-referral-assessmentreport_en.pdf. Accessed 18 Mar 2021.

18. Berry W, Daniel BS, Baker C, Foley P. Real world experience using ciclosporin in psoriasis: efficacy and toxicity in the Australasian Psoriasis Registry. Australas J Dermatol. 2020;61(4):380-2.

19. Busauschina A, Schnuelle P, van der Woude FJ. Cyclosporine nephrotoxicity. Transplant Proc. 2004;36(2 Suppl):229S-S233.

20. Boguniewicz M, Alexis AF, Beck LA, et al. Expert perspectives on management of moderate-to-severe atopic dermatitis: a multidisciplinary consensus addressing current and emerging therapies. J Allergy Clin Immunol Pract. 2017;5(6):1519-31.

21. Boguniewicz M, Fonacier L, Guttman-Yassky E, Ong PY, Silverberg J, Farrar JR. Atopic dermatitis yardstick: practical recommendations for an evolving therapeutic landscape. Ann Allergy Asthma Immunol. 2018;120(1):10-22.e2.

22. Bruin-Weller M, Biedermann T, Bissonnette R, et al. Treat-to-target in atopic dermatitis: an international consensus on a set of core decision points for systemic therapies. Acta Dermato Venereolog. 2021;101(2):adv00402. https://doi.org/10.2340/ 00015555-3751.

23. Macdonald LE, Karow M, Stevens S, et al. Precise and in situ genetic humanization of $6 \mathrm{Mb}$ of mouse immunoglobulin genes. Proc Natl Acad Sci USA. 2014;111(14):5147-52.

24. Murphy AJ, Macdonald LE, Stevens S, et al. Mice with megabase humanization of their immunoglobulin genes generate antibodies as efficiently as normal mice. Proc Natl Acad Sci USA. 2014;111(14):5153-8.

25. Gandhi NA, Pirozzi G, Graham NMH. Commonality of the IL-4/IL-13 pathway in atopic diseases. Expert Rev Clin Immunol. 2017;13(5):425-37.

26. Armstrong A, Wei W, Garcia-Horton V, et al. Risk of contraindication and drug-drug interaction (DDI) with use of systemic immunosuppressants (IMMs) in adult patients with atopic dermatitis (AD). J Invest Dermatol. 2017;137(5):S41.

27. Simpson EL, Bieber T, Guttman-Yassky E, et al. Two phase 3 trials of dupilumab vs placebo in atopic dermatitis. N Engl J Med. 2016;375(24):2335-48.

28. de Bruin-Weller $\mathrm{M}$, Thaçi D, Smith $\mathrm{CH}$, et al. Dupilumab with concomitant topical corticosteroid treatment in adults with atopic dermatitis with an inadequate response or intolerance to ciclosporin A or when this treatment is medically inadvisable: a placebo-controlled, randomized phase III clinical trial (LIBERTY AD CAFÉ). $\mathrm{Br}$ J Dermatol. 2018;178(5):1083-1101.

29. Blauvelt A, de Bruin-Weller M, Gooderham M, et al. Long-term management of moderate-to-severe atopic dermatitis with dupilumab and concomitant topical corticosteroids (LIBERTY AD CHRONOS): a 1-year, randomised, double-blinded, placebo-controlled, phase 3 trial. Lancet. 2017;389(10086): 2287-303.

30. European Medicines Agency. DUPIXENT® (dupilumab). Summary of product characteristics. Available from: https://ec.europa.eu/health/documents/ community-register/2019/20190801145601/anx_ 145601_en.pdf. Accessed 18 Mar 2021.

31. US Food and Drug Administration. DUPIXENT® (dupilumab). Highlights of prescribing information. https://www.accessdata.fda.gov/drugsatfda docs/label/2019/761055s014lbl.pdf. Accessed $1 \overline{8}$ Mar 2021.

32. Drucker A, Wang A, Li W, Sevetson E, Block JK, Qureshi AA. The burden of atopic dermatitis: summary of a report for the National Eczema Association. J Invest Dermatol. 2017;137(1):26-30.

33. Lifschitz C. The impact of atopic dermatitis on quality of life. Ann Nutr Metab. 2015;66(Suppl 1): 34-40. 
34. Ariëns LFM, Gadkari A, van Os-Medendorp H, et al. Dupilumab vs cyclosporine for the treatment of moderate-to-severe atopic dermatitis in adults: indirect comparison using the Eczema Area and Severity Index. Acta Derm Venereol. 2019;99(10): 851-7.

35. Jang DH, Heo SJ, Jung HJ, Park MY, Seo SJ, Ahn JY. Retrospective study of dupilumab treatment for moderate to severe atopic dermatitis in Korea: efficacy and safety of dupilumab in real-world practice. J Clin Med. 2020;9(6):1982.

36. Ariëns LF, van der Schaft J, Spekhorst LS, et al. Dupilumab shows long-term effectiveness in a large cohort of treatment-refractory atopic dermatitis patients in daily practice: 52 -weeks results from the Dutch BioDay registry. J Am Acad Dermatol. 2021;84(4):1000-9.

37. Spekhorst LS, Ariëns LFM, van der Schaft J, et al. Two-year drug survival of dupilumab in a large cohort of difficult-to-treat adult atopic dermatitis patients compared to cyclosporine $\mathrm{A}$ and methotrexate: results from the BioDay registry. Allergy. 2020;75(9):2376-9.

38. Fargnoli MC, Esposito M, Ferrucci S, et al. Real-life experience on effectiveness and safety of dupilumab in adult patients with moderate-to-severe atopic dermatitis. J Dermatolog Treat. 2019. https://doi.org/10.1080/09546634.2019.1682503.

39. Giavina-Bianchi M, Rizzo L, Giavina-Bianchi P. Severe atopic dermatitis: Dupilumab is not just safer, but more efficient. Allergol Immunopathol (Madr). 2020;48(6):792-7.

40. Ferrucci S, Casazza G, Angileri L, et al. Clinical response and quality of life in patients with severe atopic dermatitis treated with dupilumab: a singlecenter real-life experience. J Clin Med. 2020;9(3): 791.

41. Beck LA, Thaçi D, Deleuran M, et al. Dupilumab provides favorable safety and sustained efficacy for up to 3 years in an open-label study of adults with moderate-to-severe atopic dermatitis. Am J Clin Dermatol. 2020;21(4):567-77.

42. Merola JF, Sidbury R, Wollenberg A, et al. Dupilumab prevents flares in adults with moderate-tosevere atopic dermatitis in a 52-week randomized controlled phase 3 trial. J Am Acad Dermatol. 2021;84(2):495-7. 